\title{
A qualitative study of provider perspectives on the barriers to contraceptive use in Kaliro and Iganga Districts, Eastern Central Uganda
}

\author{
Constance Sibongile Shumba ${ }^{a}$, Jonathan Miyonga ${ }^{b}$, Judith Kiconco ${ }^{c}$, Patrick \\ Kerchan $^{\mathrm{d}}$, Tonny Tumwesigye ${ }^{\mathrm{e}}$ \\ ${ }^{a}$ Research and Grants Manager, Uganda Protestant Medical Bureau, Uganda \\ ${ }^{\mathrm{b}}$ Monitoring and Evaluation Specialist, Uganda Protestant Medical Bureau, Uganda \\ ${ }^{\text {c } F a m i l y ~ P l a n n i n g ~ O f f i c e r, ~ U g a n d a ~ P r o t e s t a n t ~ M e d i c a l ~ B u r e a u, ~ U g a n d a ~}$ \\ ${ }^{d}$ Head of Programmes, Uganda Protestant Medical Bureau, Uganda \\ ${ }^{\mathrm{e}}$ Executive Director, Uganda Protestant Medical Bureau, Uganda
}

\section{Abstract}

Background: Family planning confers unique benefits including preventing unintended pregnancies, improving maternal and child health outcomes, and increasing women's access to education and economic opportunities. However, Uganda has a low contraceptive prevalence rate of only $30 \%$, and progress in improving maternal and child health outcomes is slow.

Objective: This assessment explores community health workers' and facility-based health workers' qualitative perspectives on the use of contraceptives in the Iganga and Kaliro districts in Eastern Central Uganda.

Methods: The baseline assessment used a qualitative approach with a focused sample of community- and facility-based health workers aged 20-60 years. Two focus group discussions with Community Health Workers and four key informant interviews with facility-based health workers were conducted. Thematic content analysis was done manually.

Results: The main factors influencing contraceptive use in these communities were preference for large families, perceived inadequate knowledge of family planning and fear of side effects, inadequate spousal and family support, male domination and risk of violence, divorce and polygamy, inadequate human resource capacity and low motivation, and user fees.

Conclusion: The study findings suggest that there is low use of contraceptives for family planning in the Kaliro and Iganga districts in Uganda. Recommendations include developing a strong focus in exploring policy options to build the capacities of trained health workers to offer long-term methods in order to increase the availability of family planning options. Family planning interventions should increase the availability of contraceptive methods using gender-sensitive strategies, including community mobilization.

Nov 2016. Christian Journal for Global Health, 3(2): 60-71. 


\section{Introduction}

Sub-Saharan Africa (SSA) has the least contraceptive use globally of only $29 \%$. Uganda has a low contraceptive prevalence rate of $30 \%$ and is among the countries with unmet need for family planning. ${ }^{1-4}$ The vast benefits of family planning programs are not appreciated in some low income countries (LICs) with huge implications for improved maternal and child health from birth spacing and reducing the total fertility rate, and for financial and human resource allocation decisions. As a result, it is necessary to expand family planning interventions and increase access in LICs through accurate and affordable family planning information and methods.

There is a great need for family planning interventions in LICs, although they perform poorly due to limited funding and lack of enabling policy environments in most cases. ${ }^{2}$ Funding for family planning programs has declined significantly in the last two decades, in part due to the redirection of resources to the HIV epidemic which was a more pressing problem. ${ }^{5}$ However, there has been renewed interest in family planning in the last two years through initiatives such as the Family Planning 2020 and increased emphasis on family planning as part of health service integration. Family planning confers unique benefits including preventing unintended pregnancies, improved maternal and child health outcomes, and increased women's access to education and economic opportunities. ${ }^{6,7}$

The aim of this qualitative baseline assessment was to ascertain the use of family planning services; to understand the barriers to use, from the community and facility-based health workers' perspective, among communities in the Iganga and Kaliro districts; and to tailor an intervention package to address these barriers.

\section{Methods}

This baseline assessment used qualitative approaches with a targeted selection of Community Health Workers (CHWs) and facility-based health workers aged 20-60 years. A total of four key informant interviews (KIIs) with facility-based health workers and two focus group discussions (FGDs) with CHWs in the Iganga and Kaliro districts in Eastern Central Uganda were conducted. This followed the award of a pilot grant from the David and Lucile Packard Foundation to develop and test a family planning intervention package in these districts.

\section{Setting}

Data, from a targeted sample, was collected from two pilot health centers in the Kaliro and Iganga districts in Eastern Central Uganda in January 2014. The health facilities are faith-based, private-not-for-profit, and part of the Uganda Protestant Medical Bureau network comprising 278 member health facilities. More than $20 \%$ of these 278 health facilities are found in East Central Uganda. This region, in particular the Busoga region, has one of the highest fertility rates $(6.9 \%)$, which is above the national average of $6.2 \%$ for women. There is a high unmet need for family planning $(42 \%)$, greater than the overall unmet need in the country (34\%), among married women. ${ }^{3}$ Unmet need for family planning is defined as the proportion of productive women who are not using contraception, but who desire to postpone the next birth (spacing) or who wish to stop childbearing altogether (limiting), $21 \%$ and $14 \%$, respectively. ${ }^{3}$ Unmet need is high in East Central Uganda, meaning that $42 \%$ of productive women desire to postpone birth or stop childbearing. It was therefore deemed a suitable pilot area to test a family planning intervention package. Two health facilities out of the six assessed were chosen for family planning intervention as they had no existing support for family planning from other partners and showed potential to successfully implement a pilot 
intervention package. The assessment of the health facilities aimed to identify capacity strengths and challenges in order to establish capacity building priorities. It focused on four key areas: family planning services provision, infrastructure, stores management, and staff capacities in family planning service provision. The two health facilities had a combined catchment area population of 14,000 within 24 villages, each village served by a community health worker.

\section{Key informant interviews}

Four individual key informant interviews (KIIs) with two health workers from each of the two participating clinics were conducted to learn about family planning services and barriers to increasing contraceptive use within the catchment population of the selected health facilities. Three nursing assistants and one clinical officer participated in the facility interviews. All interviewers had worked for a minimum of two years at the facilities. The health workers interviewed were conveniently sampled due to their availability during the assessment. We examined service availability, current use of family planning services, and provider perspectives. The key informant interviews were conducted in English as all the health workers were proficient in the language. Interviews were tape recorded and additional notes taken on non-verbal cues.

\section{Focus Group Discussions (FGD)}

Two FGDs were convened, with 20 CHWs from the catchment area of each health facility to gather qualitative data on their perspectives on community attitudes and barriers to use of family planning services. CHWs are volunteers and have varying levels of education and training, mostly below high school, and are required to be able to read and write. Their main role is to carry out community health education, home visits, community sensitization, and also conduct community-based distribution of contraceptives and anti-malarial medicines. The Ministry of Health in Uganda is currently reviewing their eligibility criteria, and it is anticipated that all CHWs will have completed the fourth year of high school at the minimum and will receive some remuneration to motivate them. Purposive sampling was used to select participants, and explicit considerations were made to ensure that a good mix of men and women were included among the community health workers in each FGD. The participants for the FGDs were 11 men and 9 women, aged 24 to 60 years. Table 1 is a summary of the CHWs' sociodemographic characteristics.

Table 1. Community Health Workers' socio-demographic characteristics

\begin{tabular}{lcc}
\hline Characteristic & $\mathrm{N}=20$ & $\%$ \\
\hline Mean age (maximum) & & $48.8(60)$ years \\
Mean number of children (maximum) & $6(10)$ children \\
Marital status & & \\
Married & 19 & $95 \%$ \\
Single & 1 & $5 \%$ \\
Sex & & $55 \%$ \\
Male & 11 & $45 \%$ \\
Female & 9 & \\
\hline
\end{tabular}




\section{Data analysis}

The discussions were conducted in Lusoga, and the facilitators later translated and transcribed the discussions from audio recordings of the sessions into English. Transcripts from the key informant interviews and focus group discussions were read several times, guided by the research questions from the topic guide. The topic guide explored understanding of child spacing and the perceived advantages and disadvantages, decisionmaking on child spacing, methods used for child spacing in the community, perceived advantages and disadvantages, availability and accessibility of the methods, and community informational needs and strategies to improve use of family planning methods. Themes were identified post hoc through thematic content analysis by one member of the research team. ${ }^{8}$ Codes were also developed manually, and direct quotes, notes, and observations and voices were used to illustrate the findings.

\section{Ethical considerations}

The baseline assessment was conducted as part of a family planning program planning activity with the aim of informing program strategies and designing an appropriate intervention package. The assessment was approved by the Mengo Hospital Research Ethics Committee (665/2-15) and Uganda National Council for Science and Technology (SS 3772). Oral consent was obtained, both for participation and for making audio recordings of the Focus
Group Discussions. Prior to data collection, each data collector underwent training in research ethics adapted from the Family Health International curriculum. All participants were given information on the purpose of the baseline assessment, and informed consent was sought from all of them. The participants were assured that the data would not be linked back to them, and all information would be kept confidential and used only for the purposes of designing appropriate family planning interventions for the community. No identifying characteristics of participants were recorded during data collection, and audio recordings of FGDs were kept securely under lock and key after transcription. Participants were given light refreshments during the interviews, and no payments were given for participating in the study.

\section{Results}

Findings of this qualitative assessment are presented according to the different themes identified during the data analysis. These main themes were: (i) perceived inadequate knowledge of family planning and fear of side effects; (ii) preference for large families; (iii) inadequate spousal and family support; (iv) male domination and risk of violence, divorce, and polygamy; (v) inadequate human resource capacity and low motivation and; (vi) user fees. Table 2 shows the main themes.

Table 2: Main themes

\begin{tabular}{|c|c|c|}
\hline Main themes & Source Group & Exemplar quotes \\
\hline Perceived inadequate & Both CHWs and & "People in the community are not really educated on family \\
\hline knowledge of family & Facility-based health & planning." \\
\hline $\begin{array}{l}\text { planning and fear of side } \\
\text { effects }\end{array}$ & workers & $\begin{array}{l}\text { "People in the community believe that women can become infertile, } \\
\text { get cancer and complications due to family planning methods." }\end{array}$ \\
\hline $\begin{array}{l}\text { Preference for large } \\
\text { families }\end{array}$ & CHWs & $\begin{array}{l}\text { "People like large families because of the risk of remaining childless } \\
\text { in the event of accident." }\end{array}$ \\
\hline $\begin{array}{l}\text { Inadequate spousal and } \\
\text { family support }\end{array}$ & $\begin{array}{l}\text { Both CHWs and } \\
\text { Facility-based health } \\
\text { workers }\end{array}$ & $\begin{array}{l}\text { "Elderly people in the community want the young ones to have many } \\
\text { children." } \\
\text { "Most women are hindered by their husbands who stop them from } \\
\text { taking up family planning methods because they want them to produce } \\
\text { [give birth to] many children." }\end{array}$ \\
\hline
\end{tabular}


Male domination, risk of violence, divorce and polygamy
Both CHWs and Facility-based health workers
Inadequate human resource capacity and low motivation

User fees
Both CHWs and Facility-based health workers
"The husbands do not want to hear about family planning because they want many children. Very few men accept to hear about family planning. They say that when women go for family planning they do not get pregnant so they cheat on the husbands with other men but when they do not use contraceptives they do not cheat because they will be afraid to get pregnant with other men."

"Men do not like family planning and women think their husbands will leave them for other women if they use contraceptives and the husband does not agree with it."

"We do not have a doctor to do tube ligations and IUDs so we cannot offer these."

"Permanent methods are not accessible because they are not available at the health facility and there are no health workers who can do them. They require a doctor to run the procedure but they are not available."

"The community has been paying 1000-1500 UGX [40-60 US cents] for contraceptives so this deterred them from accessing family planning."

"It is not easy, you have to pay to access the service and people don't have the money."

\section{Use of current family planning services}

All health workers interviewed at the time of assessment reported that their health facilities offered family planning services: mainly progesterone only pills (POPs), combined oral contraceptives (COCs), condoms (male only), and Depot medroxyprogesterone acetate (DMPA) products. The most preferred method by clients was the male condom followed by DMPA products, POPs, and COCs. The CHWs also attested to the availability of these methods in both facilities. One facility was already offering intra-uterine devices (IUDs) and implants, while the other facility highlighted the need to provide these. Both facilities were reported to have expressed the need to provide permanent methods (tubal ligations and vasectomies) due to some demand from the community:

"We get clients who are interested only in IUDs, tube ligation, and vasectomy but we do not offer these here so they cannot get the service." Female Facility-based Health worker in KII

\section{Perceived inadequate knowledge of family planning and fear of side effects}

All the participants reported that there was inadequate community awareness about family planning services. The lack of awareness was attributed to lack of access to information, and this posed a barrier to use of family planning services and reflected the difficulties encountered by the healthcare workers in providing family planning and reproductive health. While it is the CHWs' and facility-based health workers' roles to conduct community education, in reality there are hindrances to doing this, including financial constraints to conduct community outreaches and lack of incentives to motivate CHWs who currently hold a volunteer status and are therefore not remunerated.

"People in the community are not really educated on family planning." CHW in FGD and Facility-based health workers in KII

The providers also reported that myths and misconceptions were rife in the community leading to reticence in using family panning methods. These myths and misconceptions included the belief that family planning causes infertility and that the POPs and COCs and oil from condoms accumulated in the body, resulting in cancer. In addition, there was a common concern that the use of contraceptives led to congenital abnormalities.

"People in the community believe that women can become infertile, get cancer and complications due to family planning methods." Male Facility-based Health worker in KII 
"People believe in the community that pills pile up in the body, and one can be taken for an operation and that oil on condoms causes cancer for women and girls, and they are not willing to use them, because when the oil remains inside, it can cause one to die." Female CHW in FGD

"When a child is born with birth abnormalities, people blame this on use of family planning." Female CHW in FGD

A majority of the participants in both the KIIs and FGDs reported that fear of side effects, such as excessive bleeding and reduced sexual drive, deterred contraceptive use, especially the DMPA product, POPs, and COCs. Both CHWs and facilitybased health workers pointed to the fact that the fear of excessive bleeding and reduced sexual drive that would potentially affect the community members' sexual lives discouraged the use of contraceptives.

"People in the community have been worried about the side effects of family planning methods. For example, some experience excessive bleeding when on injectaplan [DMPA product]." Female

Facility-based Health worker in KII

"Pills cause dizziness and loss of sexual appetite." Male CHW in FGD

CHWs reported that community members shunned permanent methods as they believed that vasectomy would reduce their sexual drive while women would experience backache as a result of tubal ligation. This would all prevent them from enjoying sex due to the decreased sexual drive and persistent backaches.

"Men believe that vasectomy reduces their sexual drive and they cannot enjoy sex as before and women with tube-ligation complain about backache." Male CHW in FGD

\section{Preference for large families}

The CHWs also reported a general preference for large families in the community. Commonly cited reasons were that in case of negative life events such as death of children, people would still have children. Those who have less would have none if calamity befell their children.

"People like large families because of the risk of remaining childless in the event of accident." Male CHW in FGD

In addition, childbearing was seen as a race against the biological clock for women, who would reach menopause without getting the desired number of children if they opted to use family planning methods.

"One can end up with less number of the desired of children by reaching menopause before getting the desired number." Female CHW in FGD

\section{Inadequate spousal and family support}

The health workers in the KIIs cited lack of partner and family support as a barrier to family planning use. Most men were reportedly against their wives' use of contraceptives because they wanted many children and had fertility concerns if their wives used contraceptives.

"Most women are hindered by their husbands who stop them from taking up family planning methods because they want them to produce (give birth to) many children." Female Facility-based Health worker in KII

"Some husbands complain that when their wives come for family planning they become barren. For example, they say after two years when they want another child the women cannot get pregnant." Male Facilitybased Health worker in KII

The CHWs also referred to the pressure from the older community members who believe in large numbers of children and promote this within the community. Furthermore, in-laws were seen as desiring many children, and if this was not fulfilled, they put a lot of pressure on the men to marry other wives. The facility-based health workers in the KIIs also confirmed that women were pressured to have many children because of the fear that their 
husbands would be pressured by relatives to get other wives.

"Elderly people in the community want the young ones to have many children." Male CHW in FGD

"If you use family planning, there is pressure from in-laws asking your husband to marry another wife." Female CHW in FGD

\section{Male domination, risk of violence, divorce and polygamy}

The health workers in the KIIs also reported that non-use of contraceptives is used as a tool by men to keep their wives under control and prevent them from cheating. This is due to the fact that the men believe that if women use contraceptives, they will have extra-marital partners. In contrast, if they do not use contraceptives, they will be forced to be faithful, as they will not want to conceive with their extra-marital partners.

"The husbands do not want to hear about family planning because they want many children. Very few men accept to hear about family planning. They say that when women go for family planning, they do not get pregnant so they cheat on the husbands with other men. However, when they do not use contraceptives they do not cheat because they will be afraid to get pregnant with other men." Female Facility-based Health worker in KII

CHWs also reported that when women use contraceptives without their spouses' permissions, there would be a risk of violence, and out of fear, many women would not attempt this. In addition, it was also perceived that in general, if a woman disobeys the husband and uses contraceptives against his will, this would lead to the husband taking on another wife.

"When the decision on the choice of family planning methods is not taken as a couple there can be violence." Female $\mathrm{CHW}$ in FGD
"Men do not like family planning, and women think their husbands will leave them for other women if they use contraceptives and the husband does not agree with it." Male CHW in FGD.

For some women, to avoid conflict and separation, they would reportedly use contraceptives discreetly without the husband's knowledge, especially DMPA product.

"Women use injectaplan [DMPA product] method because men cannot find out that their wives are using it." Male CHW in FGD

\section{Inadequate human resource capacity and low motivation}

Facility-based health workers in the KIIs reported that health facilities lacked the qualified personnel to offer some of the family planning services such as IUDs and permanent methods that require skilled staff to conduct them. The two facilities had constrained human resource capacity since the two facilities had mostly Nursing Assistants who did a short nursing certificate course of about 3-6 months. They mainly helped with bedside nursing such as wound dressing, bathing patients, preparing trolleys for procedures, and immunizations. They also offered health education and carried out patient observations. Others, who had some training in family planning, also dispensed oral contraceptives, DMPA product, condoms, and referred clients. One facility had a clinical officer who could insert IUDs, but could not offer permanent methods due to a policy that stated that this can only be done by doctors. Clinical officers had a diploma in clinical and community medicine and headed lower level health facilities with the capacity to conduct deliveries, prescribe for basic ailments, and perform minor surgeries. They also provided all contraceptive methods except the permanent voluntary surgical methods. The CHWs in the FGDs reiterated that the lack of qualified staff to offer permanent methods was hindering access in the community. 
"We do not have a doctor to do tube ligations and IUDs, so we cannot offer these." Female Facility-based Health worker in KII

"Permanent methods are not accessible because they are not available at the health facility, and there are no health workers who can do them. They require a doctor to run the procedure, but they are not available." Male CHW in FGD

\section{User fees}

Both CHWs and facility-based health workers cited user fees in the health facilities as a barrier to the use of family planning by the community. While considerably small amounts, these fees were not affordable for these rural communities.

"The community has been paying 1000-1500 UGX [40-60 US cents] for contraceptives, so this deterred them from accessing family planning." Male Health worker in KII "It is not easy. You have to pay to access the service and people don't have the money." Male CHW in FGD

\section{Discussion}

The assessment reveals that, in general, the use of family planning in the target communities was very low. Nonetheless, the most preferred contraceptive methods from the providers' perspectives were short-term, mainly condoms, POPs, COCs, and injectables, and this could possibly be due to the fact that these methods are readily available in the communities. Similarly, a study conducted in urban Kampala found that most of the participants preferred condoms as they have limited side-effects, are cheap and accessible, and prevent sexually transmitted infections. IntraUterine Devices (IUDs) and injectables were also preferred, but their cost was reportedly prohibitive. ${ }^{9}$ In our assessment, long term methods were also preferred by some community members, although inaccessible due to lack of health provider availability and capacity in offering these. The health workers attributed the observed demand for long term methods to another agency that had been conducting occasional outreaches in the communities but had withdrawn due to funding problems.

Low knowledge and the fear of side effects of family planning also hindered contraceptive use among community members. This is consistent with findings indicating that concerns about the safety of methods continue to dominate the discussion on family planning barriers. ${ }^{10}$ In our assessment, contraceptives were blamed for loss of fertility and fears that these methods led to congenital abnormalities, reduced sexual desire, and caused back pain in women. Individual and communitywide misconceptions about the side-effects and safety of contraceptives have also been cited by providers as barriers to improved family planning use, pointing to the need for investments in community education to dispel these misconceptions. ${ }^{9,11}$

Providers reported how women were frustrated by side effects due to family planning such as excessive bleeding leading to the discontinuation of the use of contraceptives. Evidence from another study conducted in Central Uganda suggests that men are often reluctant to allow their wives to use contraceptives due to low knowledge, myths and misconceptions, preference for large families, and real or perceived side effects. ${ }^{7}$ The myths and misconceptions reveal a general lack of family planning knowledge in the community and could be based on limited experiences and secondary information. Health workers must have a heightened awareness of the cultural sensitivity and improve their capacity to understand and address myths and misconceptions of family planning in the community.

The preference for large families was driven by the concept of fatalism and uncertainty about child survival. The health workers cited that many people in the communities believe that if calamity strikes, all the children may die, so it is necessary to have a large number to prepare for such 
eventualities. In the literature explored, this was substantiated with evidence that having many children in the African society is a way of guarding against extinction of the family line; a desire to perpetuate the lineage results in large families. ${ }^{13}$ Additionally, in Uganda, patrilineal tradition promotes preference for larger families.

The CHWs and facility-based health workers also cited social and spousal disapproval as barriers to contraceptive use. These findings corroborate other studies that have shown a number of social and cultural barriers to family planning including the need for women to seek partner approval. ${ }^{7,11}$ Unmet need due to disapproval by partners, women, and significant others is high in SSA and women with low education, and residing in rural areas have high unmet need. Rural women were reportedly afraid of their partners' opposition to contraceptive use, and in such communities, contraceptive use decisions are taken by men because women usually have low education and economic status level. ${ }^{6}$ Women's empowerment is strongly linked to the ability to make independent decisions including contraception use, but in patriarchal societies with a high degree of male dominance, contraception use decisions are often made by men.

Clandestine use was reported in our study by participants and similarly the gendered power dynamic resulted in clandestine contraceptive use in women in Kenya and Mali. ${ }^{10,11,14}$ Evidence suggests that in SSA, 6-20\% of contraceptive use is done secretly by women due to unequal power relations with men. ${ }^{5}$ Male partner disapproval of family planning use has been proven to be a strong predictor of non-use of family planning methods. ${ }^{11}$ It is within this context that male engagement in family planning interventions is increasingly recognized as important by providers. This has implications for gender-sensitive approaches to family planning programs that promote safe environments and equitable decision-making.

Other authors proposed that in contexts where women use contraceptives without their partner's knowledge, there may be no yield in involving men in family planning programs in the short-term, and it might not also be appropriate to target couples as the units of intervention and analysis. ${ }^{14}$ This might explain why the CHWs in our study FGDs recommended targeting men and women separately. Family planning programs focusing on women only have been shown to be ineffective, ${ }^{7}$ and our findings suggest that women and men both need to be targeted but prefer to be targeted separately. It is therefore important to respect this and ensure that both men and women are given the necessary information without assuming that information will trickle to the men from women. Engaging men in family planning is crucial, yet developing messages that appeal to them is challenging. Other authors suggest that targeted messages which emphasize both economic and health benefits of family planning are more persuasive than those highlighting the health benefits only. ${ }^{7,15,16}$ Targeted community sensitization and education of men should underscore the benefits of family planning for the entire family. It is also important to understand the root causes as to why men object to their partners' use of contraception in order to promote mutual decision-making between couples in regard to family planning. ${ }^{14}$

Our study findings indicate that CHWs and facility-based health workers believe that men disapprove of their partners' use of family planning due to the fear that it promotes extra-marital affairs. This is consistent with findings in Kenya and Uganda suggesting that female use of contraceptives was perceived by men as promoting promiscuity. ${ }^{7}{ }^{17}$ Women's use of contraceptives was viewed as a way to be unfaithful, avoid getting pregnant in extramarital relationships, and was often associated with commercial sex and, thus, not considered suitable for married women of good standing. Fear of spousal retaliation through violence, separation, or polygamy prevents women from using contraceptives against their husbands' wishes. Similarly, most rural Ethiopian women believed that if male partners oppose contraception, 
the decision must be held in respect of their culture. $^{6}$

Inadequate human resource capacity and availability were found to be barriers to family planning use in this assessment. The facilities did not have adequately qualified health workers who could offer long-term methods. Mal-distribution of health workers has been cited as one of the health workforce challenges that impedes successful implementation of family planning programs. ${ }^{2}$ It is important to put in place the appropriate human resource capacity to provide contraceptives. ${ }^{5}$ For instance, in Uganda, only doctors can provide permanent methods, and yet, they are not available to serve at lower level units, with the implication that affordable referral mechanisms need to be strengthened for the population to access services.

The health service providers reported that cost of accessing contraceptives was a barrier for the rural communities, as the two health facilities charged a small user fee for family planning services. Other authors have also reported that many women and men in low income countries still have an unmet need for contraception due to the prohibitive cost of such commodities and provider fees for health services. ${ }^{4,10,12,18}$ It is necessary to remove user fees barriers to increase family planning service use and close the gap on the unmet need for contraception in resource constrained settings.

Low community mobilization efforts contributed to low contraceptive use in this assessment, yet the benefits of community mobilization in raising awareness on service availability are indisputable. ${ }^{19}$ Sustained community mobilization has potential to improve use of family planning methods and should aim at increasing access to those most in need whilst also addressing the underlying norms that impede progress.

\section{Limitations}

The main limitation of the study is that the perspectives of women and men with unmet need for contraception were not examined, as the study focused on the perspectives of the facility-based healthcare providers and community health workers. However, we do not feel that this is likely to significantly impact the findings of this study as CHWs were chosen from among the community members. They have a deep knowledge and understanding of the health needs of their communities and, thus, hold a strong insider position. Limited generalizability to other villages in Uganda may also be a limitation as there are different cultural belief systems and capacities among healthcare facilities in other districts.

\section{Conclusion}

The study findings suggest that there is need to reduce the barriers identified from the CHWs' and facility-based health workers' perspectives that hinder increased use of family planning services in the two districts. Overall, our findings revealed that providers perceive that there is low use of family planning methods in the communities owing to social determinants and beliefs among men and women. There is need to increase community sensitization efforts to create demand. In the long term, it will be important to build the capacities of clinical officers to offer long-term methods in order to increase the availability of family planning options. Based on the findings, it was proposed that the project intervene by offering family planning camps in the communities with qualified doctors hired for the specific periods to make these family planning options accessible. Access to family planning could also be improved through removal of user fees that impede access to services within the faith-based health facilities. The faith-based health facilities obtain small grants from the government for primary health care and donations from well-wishers, but these are not sufficient to sustain the services they offer. Therefore, they usually charge small user fees, although some very poor communities are not able to afford these fees. This often puts the health facilities in a difficult position as they rely on user fees to continue service provision. 
The proposed project intervention was to provide family planning services without levying fees on clients and all family planning commodities were provided free of charge to the health facilities. The project proposed implementation of community and interpersonal education interventions to address underlying socio-cultural norms over time. This was done in recognition of the need for health service providers to promote gender-sensitive ways of engaging communities and addressing the underlying gender disparities that affected decisionmaking abilities of women with regard to family planning, including mutual discussions on family planning between spouses. This is a major cultural intervention which will ultimately take a great deal of time to achieve. The proposed intervention to reduce unmet need for family planning and improve maternal and child health outcomes was also to implement a package to strengthen capacity of religious leaders. This involved equipping them with knowledge on health timing and spacing of pregnancies and providing guidance on key messages they can incorporate in their sermons advocating for use of FP in their communities as well as providing counselling and referral support to community and facility-based health workers.

\section{References}

1. Population Reference Bureau. 2014 World Population Data Sheet [Internet]. Available from: http://www.prb.org/pdf14/2014-world-population-datasheet_eng.pdf [Cited 2016 June 5]

2. Prata N. Making family planning accessible in resource-poor settings. Philos T Roy Soc B. 2009;364(1532):3093-9. http://doi.org/10.1098/rstb.2009.0172

3. Uganda Bureau of Statistics (UBOS) and ICF International Inc. Uganda demographic and health survey 2011. Kampala, Uganda: UBOS and Calverton, Maryland: ICF International Inc. 2012.

4. Population Reference Bureau. Unmet need for family planning [Fact Sheet]. 2012. Available from:
http://www.prb.org/Publications/Datasheets/2012/worldpopulation-data-sheet/fact-sheet-unmet-need.aspx [Cited 2016 June 5]

5. Bongaarts J, Sinding SW. A response to critics of family planning programs. Int Perspec Sex Repro Health. Mar 2009;35(1).

6. Bogale B, Wondafrash M, Tilahun T, Girma E. Married women's decision making power on modern contraceptive use in urban and rural Southern Ethiopia. BMC Public Health. 2011;11(342).

http:/dx.doi.org/10.1186/1471-2458-11-342

7. Kabagenyi A, Jennings L, Reid A, Nalwadda G, Ntozi J, Atuyambe L. Barriers to involvement in contraceptive uptake and reproductive health services: a qualitative study of men and women's perceptions in two rural districts in Uganda. Reproductive Health.

2014;11(21) http://dx.doi.org/10.1186/1742-4755-11-21.

8. Braun V, Clarke V. Using thematic analysis in psychology. Qual Research Psych. 2006;3(2):77-101. [ISSN1478-0887].

http://dx.doi.org/10.1191/147888706qp063oa Available from: http://eprints.uwe.ac.uk/11735 [Cited 2016 May 23].

9. Wanyenze RK, Wagner GJ, Tumwesigye NM, Nannyonga M, Wabwire-Mangen F, Kamya MR. Fertility and contraceptive decision-making and support for HIV-infected individuals: client and provider experiences and perceptions at two HIV clinics in Uganda. BMC Public Health. 2013;13(98). http://dx.doi.org/10.1186/1471-2458-13-98

10. Harrington EK, Newman SJ, Onono M, Schwartz KD, Bukusi AE, Cohen CR et al. Fertility intentions and interest in integrated family planning services among women living with HIV in Nyanza Province, Kenya: a qualitative study. Infect Dis Obstet Gynecol. 2012;809682. http://doi.org/10.1155/2012/809682

11. Newmann SJ, Mishra K, Onono M, Bukusi EA, Cohen CR, Gage O, et al. Providers' perspectives on provision of family planning to HIV-Positive individuals in HIV Care in Nyanza Province, Kenya. AIDS Res Treat. 2013;2013(915923). http://dx.doi.org/10.1155/2013/915923 
12. Graffy J, Goodhart C, Sennet K, Kamusiime G, Tukamushaba H. Young people's perspectives on the adoption of preventive measures for HIV/AIDS, malaria and family planning in South-West Uganda: focus group study. BMC Public Health. 2012;12(1022).

http://dx.doi.org/10.1186/1471-2458-12-1022

13. Makinwa-Adebusoye P; Population Division. Department of Economic and Social Affairs. United Nations Secretariat. Socio-cultural factors affecting fertility in Sub-Saharan Africa. Workshop on prospects for fertility decline in high fertility countries. New York; 2001 July 9-11.

14. Castle S, Konate MK, Ulin PR, Martin S. A qualitative study of clandestine contraceptive use in urban Mali. Stud Family Plan. 1999 Sept;30(3):241-8. http://dx.doi.org/10.1111/j.1728-4465.1999.00231.x

15. Kululanga LI, Sunby J, Malata A, Chirwa E. Striving to promote male involvement in maternal healthcare in rural and urban settings in Malawi- a qualitative study. Health. 2011;

http://dx.doi.org/10.1186/1742-4755-8-36

16. Shattuck D, Kerner B, Gilles K, Hartmann M, Ng'ombe T, Guest G. Encouraging contraceptive uptake by motivating men to communicate about family planning: the male motivator project. Am J Public Health. June 2011;101(6):1089-95.

http://dx.doi.org/10.2105/AJPH.2010.300091

17. Kenya National Bureau of Statistics and ICF Macro. Kenya demographic and health survey 2008-09.

Calverton, Maryland: KNBS and ICF Macro. 2010.

18. Mutyaba T, Faxelid E, Mirembe F, Weiderpass E. Influences on uptake of reproductive health services in Nsangi community of Uganda and their implications for cervical cancer screening. BMC Repro Health. 2007;4(4). http://dx.doi.org/10.1186/1742-4755-4-4

19. Gazi R, Hossain SS, Zaman K, Koehlmoos TP. Community mobilization for safe motherhood. Cochrane DB Syst Rev. 4 (2011): CD009091

Peer Reviewed

Competing Interests: None declared.

Acknowledgments: This baseline assessment was funded by the David and Lucille Packard Foundation through Africa Christian Health Associations Platform (ACHAP) for a pilot project to which Uganda Protestant Medical Bureau was a sub-grantee in the two baseline assessment districts.

Author contributions: CS designed the study while JM, PK, TT and JK gave input into the tools. CS, JK, and JM were responsible for data collection, coding, and analysis. CS wrote the draft manuscript. JM, JK, PK, and TT critically reviewed the draft, adding substantial intellectual content.

Correspondence: Constance Sibongile Shumba, Uganda Protestant Medical Bureau. konstansezw@gmail.com

Cite this article as: Shumba SC, Miyonga J, Kiconco J, Kerchan P, Tumwesigye T. A qualitative study on provider perspectives on the barriers to contraceptive use in Kaliro and Iganga districts, Eastern Central Uganda. Christian Journal for Global Health (Nov 2016), 3(2):49-60.

(C) Shumba SC, Miyonga J, Kiconco J, Kerchan P, Tumwesigye T. This is an open-access article distributed under the terms of the Creative Commons Attribution License, which permits unrestricted use, distribution, and reproduction in any medium, provided the original author and source are properly cited. To view a copy of the license, visit http://creativecommons.org/licenses/by/4.0/

\section{www.cjgh.org}

Nov 2016. Christian Journal for Global Health, 3(2):60-71. 